\title{
Corrigendum: Excess aldosterone under normal salt diet induces cardiac hypertrophy and infiltration via oxidative stress
}

Kaoru Yoshida, Shokei Kim-Mitsuyama, Ryotaro Wake, Yasuhiro Izumiya, Yasukatsu Izumi, Tokihito Yukimura, Makiko Ueda, Minoru Yoshiyama and Hiroshi Iwao

Hypertension Research (2016) 39, 482; doi:10.1038/hr.2016.33

Correction to: Hypertension Research (2005) 28, 447-455; doi:10.1291/ hypres. 28.447

It has come to the authors' attention that three representative images used in Figure 2(B) of this article were incorrect. The figure with correct representative images is presented below. This correction does not affect the results or the conclusions of this manuscript.
(B)
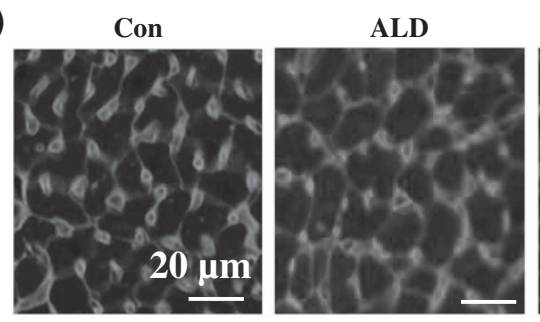

ALD / Tem
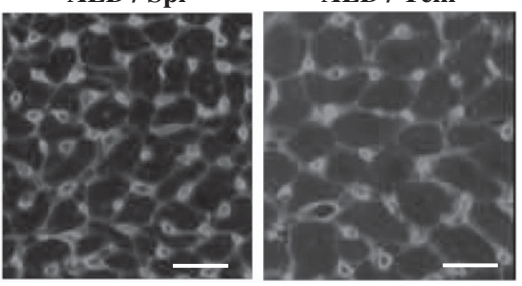

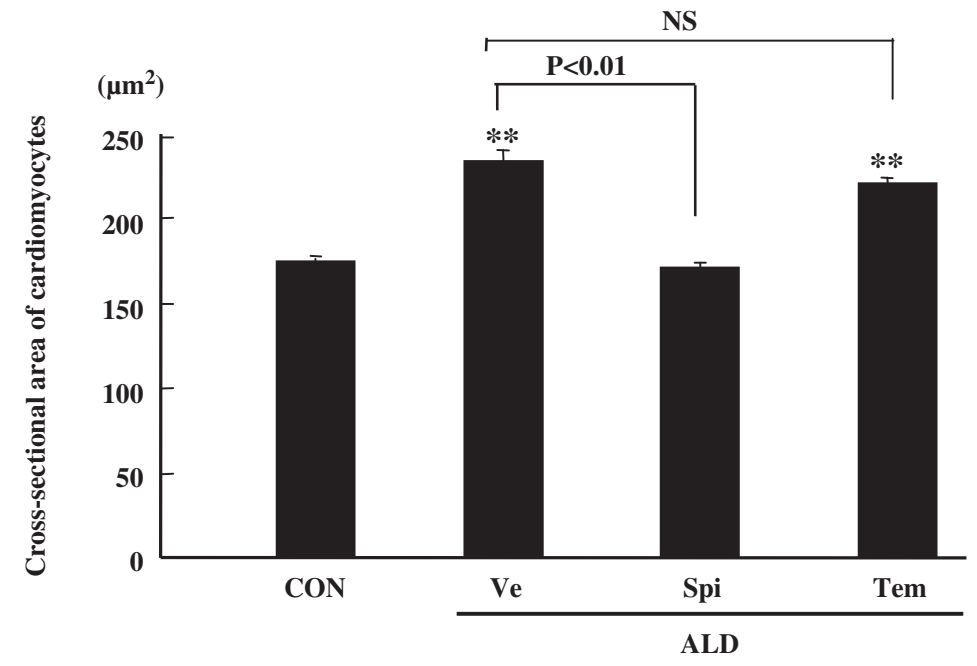

Figure 2 A full color version of this figure is available at the Hypertension Research journal online. 